\title{
A SAÚDE DO TRABALHADOR EM RADIOLOGIA: ALGUMAS CONSIDERAÇÕES ${ }^{1}$
}

\author{
Cátia Inácia Brand², Rosane Teresinha Fontana ${ }^{3}$ Antônio Vanderlei dos Santos ${ }^{4}$
}

\footnotetext{
${ }^{1}$ Artigo original apoiado pelo Grupo de Pesquisa em Enfermagem, Saúde e Educação da Universidade Regional Integrada do Alto Uruguai e das Missões (URI) - Campus Santo Ângelo - RS.

${ }^{2}$ Acadêmica do $9^{\circ}$ semestre do Curso de Enfermagem da URI - Campus Santo Ângelo - RS. Rio Grande do Sul, Brasil. E-mail: catiabrand@yahoo.com.br

${ }^{3}$ Doutoranda em Enfermagem pela Universidade Federal do Rio Grande do Sul. Professora da graduação e pós-graduação latu sensu na URI - Campus Santo Ângelo - RS. Rio Grande do Sul, Brasil. E-mail: rfontana@urisan.tche.br

${ }^{4}$ Doutor em Ciências. Professor da graduação e pós-graduação scrictu sensu na URI - Campus Santo Ângelo - RS. Rio Grande do Sul, Brasil. E-mail: vandao@urisan.tche.br
}

RESUMO: Estudo exploratório, com desenho transversal, que teve como objetivo identificar sinais e sintomas autorreferidos por profissionais que trabalham em serviços de radiologia e os conhecimentos acerca da radioproteção e legislação. Os dados foram coletados junto a oito técnicos em radiologia que trabalham em três serviços localizados na região noroeste do estado do Rio Grande do Sul, utilizando-se um questionário autoaplicável e analisados mediante estatística descritiva. A cefaleia foi o sintoma mais citado pelos trabalhadores. Observou-se deficiência de saberes sobre legislação e uso de equipamentos de proteção individual. Acredita-se que a valorização no trabalho inicia com ações que privilegiem a segurança ocupacional, de modo a prevenir agravos e promover a saúde do trabalhador.

DESCRITORES: Saúde do trabalhador. Radiologia. Sinais e sintomas.

\section{THE HEALTH OF RADIOLOGY WORKERS: SOME CONSIDERATIONS}

ABSTRACT: This exploratory study using cross-sectional design aimed to identify self-reported signs and symptoms by professionals working in radiology services and their knowledge concerning radiation protection and legislation. Data was collected from eight radiology technicians who work in three offices located in the northwestern state of Rio Grande do Sul, Brazil, using a self-administered questionnaire and analyzed using descriptive statistics. Headache was the symptom most often cited by workers. This study observed deficiencies of knowledge concerning laws and usage of personal protective equipment. It is believed that proper valuation at work begins with actions which promote occupational safety in order to prevent injuries and promote worker health.

DESCRIPTORS: Occupational health. Radiology. Signs and symptoms.

\section{LA SALUD DEL TRABAJADOR EN RADIOLOGÍA: ALGUNAS CONSIDERACIONES}

\begin{abstract}
RESUMEN: Se trata de un estudio exploratorio, con diseño transversal, que tuvo como objetivo identificar los signos y los síntomas reportados por los profesionales que trabajan en los servicios de radiología y sus conocimientos sobre la protección radiológica y la legislación. Los datos fueron recolectados a partir de ocho técnicos de radiología que trabajan en tres oficinas ubicadas en la región noroeste del estado de Rio Grande do Sul - Brasil. Se utilizó un cuestionario auto-administrado, y los datos se analizaron mediante estadística descriptiva. La cefalea fue el síntoma más frecuentemente citado por los trabajadores. Se observó la falta de conocimientos sobre la legislación y el uso del equipo de protección personal. Se cree que la valoración del trabajo se inicia con acciones que privilegien la seguridad ocupacional, para prevenir lesiones y promover la salud de los trabajadores.
\end{abstract}

DESCRIPTORES: Salud laboral. Radiología. Signos y síntomas. 


\section{INTRODUÇÃO}

Em decorrência de uma demanda de atendimentos a pacientes com necessidades cada vez mais complexas, é exigido do setor saúde a incorporação de novas tecnologias e formas de organização do trabalho. Modernizou-se o diagnóstico e a terapêutica e o cuidado exibe uma conformação caracterizada pelo atendimento de um sujeito também complexo. Porém, esta nova configuração pode contribuir para o adoecimento decorrente da exposição insegura a riscos ocupacionais.

Os fatores de risco ocupacionais, de acordo a Organização Pan-Americana de Saúde (OPAS), ${ }^{1}$ são classificados em químicos, biológicos, ergonômicos, psicossociais, mecânicos, de acidentes e físicos. Os agentes físicos englobam diversas formas de energia a que possam estar expostos os profissionais, tais como vibração, pressão, ruído, temperaturas extremas, radiações não ionizantes e radiações ionizantes, sendo esta última o objeto deste estudo.

Optou-se por estudar a relação entre o trabalho e a saúde junto aos técnicos em radiologia, tendo como foco a exposição ao risco físico a que mais estão sujeitos, ou seja, à radiação ionizante. Considerou-se a relativa escassez de pesquisas acerca do tema, a fim de contribuir para a promoção da saúde destes trabalhadores. São doenças decorrentes da exposição a radiações ionizantes: neoplasias, síndromes mielodisplásicas, anemia aplástica, púrpura e outras manifestações hemorrágicas, agranulocitose e outros transtornos especificados dos glóbulos brancos; polineuropatia induzida pela radiação; blefarite, conjuntivite, catarata, pneumonite, fibrose pulmonar, gastroenterite e colite tóxica, radiodermatite e outras afecções da pele e do tecido conjuntivo, infertilidade masculina, entre outras. ${ }^{1}$

Sabe-se que a identificação ou comprovação de efeitos da exposição ocupacional a fatores ou situações de risco, pode exigir, além da história ocupacional e dos dados epidemiológicos compatíveis com a hipótese do dano, a complementação diagnóstica por meio da realização de exames complementares específicos, quais sejam toxicológicos, eletromiográficos, de imagem, clínicos, entre outros. ${ }^{1}$

O nexo causal entre a exposição e o adoecimento é um processo complexo; porém acredita-se que estudos desta natureza possam contribuir para que os próprios trabalhadores motivem-se a buscar estes nexos, o que se constitui como fator preditivo na prevenção de agravos decorrentes do trabalho.

Técnicos em radiologia trabalham sob condições inadequadas, do ponto de vista de segurança, tais como a falta de sinalização indicando a utilização de radiação, ausência de vidro plumbífero, EPIs insuficientes em quantidade e especificidade e desatenção às precauções padrão, ${ }^{2-3}$ o que é preditivo de agravos à saúde do trabalhador. Assim como os profissionais técnicos, estudantes de odontologia demonstraram alguma negligência no uso de EPIs e nas condutas de biossegurança em relação ao contato com a radiação, conforme apontado num estudo realizado na Paraíba. ${ }^{4}$

Considerando que muitos técnicos desempenham suas funções em ambiente sem renovação adequada do ar, com sistemas deficientes de ventilação, preparam soluções e revelam radiografias, sem EPI adequado, entre outros riscos físicos, químicos, ergonômicos e psicossociais, este estudo reveste-se de relevância, na medida em que busca explorar morbidades que acometem estes trabalhadores. ${ }^{5}$

A norma brasileira de proteção radiológica da Comissão Nacional de Energia Nuclear (BR), além de definir parâmetros sobre a produção, o armazenamento de materiais e a prática que envolve as radiações ionizantes, também estabelece requisitos básicos ao trabalho seguro dos profissionais. Entre outras recomendações, um dos princípios prescritos nas Diretrizes Básicas de Radioproteção refere-se às doses (quantidades de radiação) individuais de trabalhadores que utilizam materiais radioativos, os quais não devem exceder os limites estabelecidos na Norma CNEN-NE-3.01. ${ }^{6}$ Os empregadores dos indivíduos ocupacionalmente expostos são responsáveis pela otimização da radioproteção, e estes devem seguir as recomendações de segurança.

Isto posto, o uso inadequado e a exposição desnecessária à radiação ionizante são responsáveis por inúmeros danos ao organismo vivo. Porém, todos estes agravos podem ser evitados ou prevenidos, considerando que se trata de riscos e, como tais, representam probabilidades e não certezas.

Tem-se em vista o interesse dos pesquisadores na área de saúde do trabalhador como uma disciplina envolvida com a articulação de meios para a prevenção de agravos decorrentes do trabalho, assim como em ações de promoção, reabilitação e vigilância em saúde e sabe-se dos 
danos que a radiação ionizante pode causar à saúde dos sujeitos que trabalham diretamente expostos a ela. Sendo assim, o objetivo deste estudo foi identificar sinais e sintomas autorreferidos por profissionais que trabalham em serviços de radiologia e os conhecimentos acerca da radioproteção e legislação, de modo a alicerçar estratégias para a melhoria da qualidade de vida e saúde deste trabalhador, considerando que, a partir da exploração semiológica e de outros saberes relativos, pode-se investir em educação.

É válido salientar que a inserção de enfermeiros e acadêmicos de enfermagem na investigação de determinantes do processo saúde-doença contribui para a formação de profissionais de saúde, em condições de pensar e agir como sujeitos sociais envolvidos com a democracia e a emancipação humana que a sociedade contemporânea exige. É uma estratégia que pode desenvolver habilidades, possibilitando a transformação dos saberes e práticas com vistas à atenção à saúde da comunidade, da mesma forma em que oportuniza a percepção da complexidade de suas práticas e a articulação de conhecimentos para a (re)invenção dos modos de lidar com a realidade de saúde. ${ }^{7}$

\section{MATERIAIS E MÉTODOS}

Trata-se de uma pesquisa exploratória, com desenho transversal. Não foi intenção associar variáveis, apenas explorar possíveis morbidades e riscos ocupacionais a que estão expostos estes trabalhadores, bem como seus conhecimentos. Este tipo de pesquisa pode ser usada para identificar grupos de risco e peculiaridades de uma comunidade para melhor direcionar as necessárias intervenções. ${ }^{8}$

A população em estudo era de 16 profissionais, sendo que todos os técnicos em radiologia que trabalhavam em três hospitais que possuem serviço de radiologia, na região de abrangência de uma Coordenadoria Estadual de Saúde, situados no interior do estado do Rio Grande do Sul.

A amostra foi composta dos oito técnicos em radiologia que aceitaram participar do estudo. Técnico em radiologia, de acordo com a lei $\mathrm{N}^{\mathrm{O}}$ 7394 de 29 de outubro de $1985^{\circ}$, são profissionais que executam técnicas radiológicas, no setor de diagnóstico; radioterápicas, no setor de terapia; radioisotópicas, no setor de radioisótopos e de medicina nuclear, além de operações correlatas em indústrias. Neste estudo, participaram somente operadores de máquinas de raio $\mathrm{X}$.
Sabe-se da limitação de amostras pequenas à generalização; porém, como se trata de um estudo exploratório inicial, acredita-se que o número de sujeitos permitiu atingir os objetivos e evidenciou, mesmo que parcialmente, a realidade configurada na região.

Os sujeitos foram convidados a participar da pesquisa a partir da explicitação acerca dos propósitos da mesma. Foram critérios de inclusão propostos: trabalhar operando equipamentos de raio $\mathrm{X}$ das respectivas instituições e aceitar participar da pesquisa, mediante assinatura de um Termo de Consentimento Livre e Esclarecido. Foram critérios de exclusão estar em férias ou em licença e trabalhar em outro setor do serviço de radiologia, que não o de operador de máquinas de raio $X$.

Para a coleta dos dados foi utilizado um questionário autoaplicável, mediante agendamento com os sujeitos, com a presença do pesquisador, contendo 30 perguntas semiestruturadas que versaram sobre sinais e sintomas sugestivos de dano ou adoecimento por contato com radiações ionizantes e conhecimentos acerca da segurança do trabalhador no setor.

O tratamento dos dados deu-se a partir da análise da distribuição da frequência das variáveis, utilizando-se a estatística descritiva, com apoio do software Excel Windows, versão 2007. A análise de dados transversais supõe a interpretação dos resultados obtidos através de uma estatística descritiva para classificar e analisar os mesmos, tendo como base a frequência relativa. ${ }^{8}$

A pesquisa respeitou a Resolução 196/96 do Conselho Nacional de Saúde. ${ }^{10} \mathrm{O}$ estudo obteve parecer favorável do Comitê de Ética da Universidade Regional Integrada do Alto Uruguai e das Missões (URI) Campus de Santo Ângelo-RS, sob protocolo No 074-04/PPH/09, bem como a autorização dos gestores dos hospitais em estudo.

\section{RESULTADOS E DISCUSSÃO}

\section{Caracterização dos sujeitos}

Participaram da pesquisa um total de oito técnicos em radiologia, sendo três do sexo feminino e cinco do sexo masculino. Todos possuem uma carga horária de quatro horas de trabalho, sendo que dois dos participantes acumulam mais uma jornada de trabalho em outra instituição. $\mathrm{O}$ artigo 14 da lei que regulamenta a profissão de técnico em radiologia prevê jornada de trabalho de $24 \mathrm{~h}$ 
semanais, o que se pressupõe que a mesma está adequada à legislação neste quesito. ${ }^{9}$

Com relação ao tempo de serviço em radiologia, há profissionais que desempenham a função há vários anos como também há outros que estão há poucos meses no desempenho da atividade. Os dados encontrados foram os seguintes: 12,5\% trabalham nesta ocupação há 30 anos; 37,5\% de 10 a 17 anos e $50 \%$ trabalham de quatro a menos de um ano.

\section{Sintomas/sinais autorreferidos}

A sintomatologia apresentada pelos técnicos em radiologia contemplou apenas o período referente ao início de suas atividades até o momento da realização do estudo. Sendo assim, foram encontrados os seguintes dados relativos aos sintomas e sinais: dentre os participantes, $62,5 \%$ referiram ter pelo menos uma vez ao mês sensação de apetite diminuído. Com relação a náuseas, $37,5 \%$ dos pesquisados mencionaram ter uma vez ao mês esta sensação. Quanto ao vômito, 12,5\% referiram ter uma vez a manifestação desse sinal; os demais afirmaram não apresentá-lo. Quanto à diarreia, 37,5\% perceberam-na uma vez no mesmo período. Com relação ao cansaço e dor muscular, $12,5 \%$ dos pesquisados responderam apresentar essa sensação durante o mês.

A cefaleia foi um dos sintomas prevalentes. Os dados encontrados demonstram que $12,5 \%$ dos técnicos em radiologia a apresentam uma vez ao mês; $12,5 \%$ duas vezes, $12,5 \%$ cinco vezes, $25 \%$ referem mais de cinco episódios por mês e $25 \%$ referiu não ter dores de cabeça habitualmente.

Um estudo feito junto a 168 trabalhadores da equipe de enfermagem de uma comunidade hospitalar concluiu que $98 \%$ destes sujeitos referiram apresentar este sintoma no cotidiano de sua ocupação. As dores de cabeça, embora comuns, podem intervir negativamente no desempenho do trabalho, considerando que a dor limita e produz sofrimento. ${ }^{11}$

\section{Doenças autorreferidas}

Ao serem indagados sobre como consideram suas condições de saúde, $62,5 \%$ referiram sentirem-se em ótimas condições. Esse resultado complementa o dado sobre a frequência de adoecimento no período de um ano, no qual 100\% dos profissionais responderam que não adoecem facilmente, o que representa um dado animador, considerando-se a grande exposição ao risco físico a que se sujeitam por conta de sua ocupação; porém, não afasta a preocupação, tendo em vista que muitos efeitos insurgem após muitos anos de atividade.

Denominam-se Efeitos Estocásticos quando os danos são proporcionais à dose recebida e pode levar até quarenta anos para ser detectado, como no caso de neoplasias e Efeitos Determinísticos, quando as consequências do dano aumentam conforme a dose absorvida e são produzidas por doses elevadas. Neste caso, o dano é previsível. Além disso, classificam-se os danos em Efeitos Imediatos que podem ocorrer até poucas semanas ou até mesmo dias da exposição e os Efeitos Tardios, que podem levar anos para aparecer no ser humano. ${ }^{6}$

Com relação às patologias apresentadas por esses trabalhadores, tais como anemias, quadros hemorrágicos graves, plaquetopenia, doenças medulares, lesões de pele com características cancerígenas e doenças pulmonares graves, entre outras, constantes na Lista de Doenças Relacionadas ao Trabalho do Ministério da Saúde ${ }^{1}$, percebeu-se que, na sua grande maioria, os resultados foram negativos. Apenas com relação ao quadro respiratório, obteve-se um dado diferente, pois $12,5 \%$ dos técnicos em radiologia referiram já ter tido quadros de pneumonia grave. Pneumonite por radiação é citada na lista de doenças relacionadas com agente ou fator de risco de natureza ocupacional por exposição à radiação ${ }^{1}$, denominadas e codificadas segundo o Código Internacional de Doenças.

Um resultado que chamou a atenção é que um dos participantes da pesquisa respondeu ter tido dois filhos com alterações genéticas. Ao ser indagado sobre o assunto, preferiu não entrar em detalhes, o que foi respeitado.

Pode-se inferir, a partir desses dados, que os trabalhadores pesquisados, aparentemente, são saudáveis, o que não descarta a probabilidade de adoecimento insidioso, característico das ocupações desta natureza. Daí a necessidade de investigações clínicas frequentes acerca de suas funções vitais e de exames laboratoriais periódicos.

\section{Rotina de exames laboratoriais}

Dentre os vários exames que avaliam o estado de saúde, foi questionado aos técnicos em radiologia sobre três exames básicos de rotina que devem ser realizados para o acompanhamento da situação de saúde. Trata-se dos exames que, 
periodicamente, os trabalhadores de radiologia expostos às radiações ionizantes devem realizar, quais sejam o hemograma completo e a contagem de plaquetas, feitas não só na admissão ao serviço, como também semestralmente. ${ }^{12}$

Quando indagados se já haviam realizado exames que avaliam a função leucocitária e plaquetária, desde a sua admissão no trabalho em radiologia, as respostas encontradas foram unânimes; todos responderam que já fizeram esses exames e que nenhum dos resultados apresentou alteração.

Em contrapartida, o dado revelado em relação aos exames da função eritrocitária é desanimador. Cerca de $25 \%$ do grupo de técnicos pesquisados responderam nunca ter realizado esse exame desde que ingressaram na radiologia, o que é prevalente entre os que se encontram há mais tempo no serviço. Esse dado permite uma reflexão sobre a causa desta negligência: ou os sujeitos não estão sendo orientados sobre a relevância desse controle ou só realizarão os exames quando já apresentarem algum sinal e/ ou sintoma de adoecimento.

Segundo o Programa de Controle Médico de Saúde Ocupacional (PCMSO) ${ }^{12}$ os empregadores dos serviços de radiologia e diagnósticos por imagem são responsáveis por implantar a realização de exames periódicos de saúde ocupacional que tem como objetivo principal a prevenção, rastreamento e diagnóstico de danos à saúde relacionados ao trabalho.

Isto posto, não basta ter conhecimento da existência da legislação, de normas de proteção à saúde do trabalhador para garantir a segurança numa empresa, é necessário que as ações apontadas por aquela, sejam operacionalizadas. A estruturação dos meios acerca de como esse processo será feita não é só responsabilidade do empregador, mas também dos trabalhadores que não são os únicos favorecidos dessas ações. Os usuários e gestores também o são, pois trabalhar de forma saudável garante qualidade de vida e de trabalho, bem como prestação eficiente de serviço.

\section{Uso de EPIs}

Segundo a norma regulamentadora $n^{\circ} 6$, estabelecida pelo Ministério do Trabalho e Emprego (BR) ${ }_{1}^{13}$ considera-se Equipamento de Proteção Individual (EPI), todo o equipamento usado para proteger o trabalhador de algum risco à sua integridade física. A empresa é responsável por oferecer aos seus trabalhadores, gratuitamente, os EPI necessários e recomendados, segundo o risco a que os servidores se expõem, sendo que o mesmo deve estar em perfeito estado de conservação para o uso e devidamente aprovado por órgão nacional competente. Da mesma forma, os trabalhadores devem comprometer-se a usá-los.

Dos respondentes, $100 \%$ dos participantes reconhecem a importância do uso de Equipamentos de Proteção Individual. Embora 75\% referissem que sabem usar adequadamente os EPIs, de acordo com a especificidade e/ou tipo de procedimento, $12,5 \%$ admitem não saber qual deles deve ser usado para cada situação característica de sua ocupação; $12,5 \%$ dos profissionais aludem deficiência de conhecimentos sobre estas especificidades.

Ao serem indagados sobre o uso contínuo dos EPI ao trabalharem com radiações ou entrarem em contato com fonte de emissão de radiações ionizantes, $12,5 \%$ dos entrevistados referiram não fazer o uso constante dos mesmos. Esse dado causa apreensão, especialmente porque comportamentos desta natureza contribuem para exacerbar o risco ocupacional e favorecer o sofrimento e /ou adoecimento.

São equipamentos de proteção que devem ser usados no trabalho direto à fonte de radiação: vestimentas de segurança que ofereçam proteção ao tronco e luva de segurança para proteção das mãos contra radiações ionizantes, assim como anteparos de vidro plumbífero. Além disso, considerando as atividades exercidas, são recomendados EPIs tais como luvas, máscaras e aventais de látex nitrílico para proteção contra os agentes químicos usados durante a preparação de soluções e máscaras próprias para retenção de impurezas menores do que $5 \mathrm{~m}$ contra os agentes biológicos que expõem o trabalhador durante os exames.,13

Estudos demonstram que profissionais e/ ou estudantes de alguns hospitais ou centros de saúde há negligência quanto ao uso/disponibilidade de todos os EPIs necessários ao trabalho nesta atividade. Nem todos os sujeitos que têm contato com radiação ionizante, utilizam-se de métodos de radioproteção individual tais como protetores de gônadas, de tireoide, luvas, óculos plumbíferos, biombo de proteção individual, entre outros, embora os aventais sejam usados por muitos ${ }^{2,4} \mathrm{O}$ que demonstra a necessidade de investimentos em formação acadêmica e em educação permanente em saúde, de forma a prevenir agravos.

Cabe mencionar que a radiação ionizante atua de forma lenta e causa danos à saúde quando 
as precauções para evitarem-se exposições desnecessárias não são respeitadas rigorosamente. Sendo assim, a "proteção radiológica constitui importante ferramenta na promoção da saúde dos trabalhadores que exercem suas atividades com radiação ionizante e, nesse caso, a educação permanente pode contribuir para a melhoria desse processo de trabalho". ${ }^{14: 769}$

\section{Saberes sobre legislação e normas}

Sobre as legislações que regulamentam a segurança do trabalhador em radiologia optouse por enfatizar as duas principais que legislam sobre as radiações ionizantes e a segurança do trabalhador, ou seja, a Portaria $453 / 98^{15}$ e a Norma da Comissão Nacional de Energia Nuclear (CNENNN-3.01/95). ${ }^{16}$

Quando questionados sobre os conhecimentos acerca da portaria, 453/98, 37,5\% relataram conhecê-la; os outros $62,5 \%$ dizem não saber/ lembrar nada sobre a mesma. Dos que a conhecem, $50 \%$ lembram alguma coisa de seu conteúdo; dos que não a conhecem e/ou não se lembram, reforçaram a necessidade de revê-la e reconhecem a importância disso.

Em comparação, quando o assunto foi a norma da CNEN-NN-3.01, a análise dos dados respondidos pelos técnicos em radiologia demonstrou que $75 \%$ a conhecem e $25 \%$ não. Quanto aos assuntos abordados pela referida norma, 62,5\% consideraram satisfatórios seus conhecimentos e $37,5 \%$ mencionaram não se lembrarem de nada sobre a mesma. Quanto à necessidade de estudá-la, $37,5 \%$ responderam que seria importante a prática de educação permanente sobre esse assunto e $62,5 \%$ não vislumbram a mesma seriedade.

Tais dados assinalam a lacuna existente entre os serviços, seus gestores e a regulação sanitária e/ou da saúde do trabalhador, no que tange à articulação de meios para a otimização de espaços democráticos de discussão acerca da legislação que regulamenta a radioproteção e a fiscalização disso. O estudo não teve a intenção de verificar condições de risco e/ou sanitárias do ambiente, mas vale reforçar que encontros de discussão acerca de medidas de proteção, coletiva, individual e ambiental, são temas que devem balizar todo e qualquer programa de educação permanente em saúde.

Um estudo realizado com o objetivo de averiguar que importância os técnicos de radiologia atribuem à implementação de um programa de controle de qualidade no setor, realizado em Por- tugal, concluiu que pouco mais da metade de população estudada tem conhecimento sobre programas de qualidade em radiologia e que $62,5 \%$ não sabem em que consiste um Programa de Controle de Qualidade (PCQ), o que, segundo os autores, torna difícil implementá-lo sem antes socializarem conceitos a respeito do tema. ${ }^{2}$ Note-se que, além de avaliar os equipamentos e áreas de radiação, a medição das doses individuais de radiação dos trabalhadores é um dos critérios que constituem um PCQ em radiologia, além de que programas de monitoração ocupacional podem auxiliar não só à supervisão e treinamento, mas, medindo padrões de segurança do local de trabalho, é possível verificar as possíveis exposições à radiação que os sujeitos estão sujeitos a receber. ${ }^{17}$

Analisando este contexto dado, percebe-se que o grupo de técnicos em radiologia pesquisado trabalha sob conhecimentos insuficientes à proteção de si e dos pacientes e que, portanto, a educação permanente é uma carência dos serviços. Cabe reforçar a ideia de inovar e de crescer por meio da interação dos saberes.

\section{Educação permanente em saúde e promoção da saúde do trabalhador em radiologia}

Nesta pesquisa, constatou-se que metade dos sujeitos trabalha há mais de 10 anos em contato com radiações ionizantes. Estes mesmos profissionais relataram que obtêm atualizações na sua área, principalmente por meio de busca individual; um dado que inquieta, considerando os danos causados pela exposição à radiação ionizante e pela desconsideração com a atualização que favorece a prevenção e exposições desnecessárias.

Quando questionados sobre a necessidade da prática de educação permanente em sua área de atuação profissional, as respostas obtidas denunciaram que $87,5 \%$ dos trabalhadores admitem o valor desta estratégia.

O conceito de educação permanente se materializa na possibilidade da troca de saberes após a formação inicial. A partir do reconhecimento da realidade vivenciada pelos sujeitos em seu local de trabalho e, constatando-se as deficiências de saberes e fazeres para o adequado desempenho da função busca-se, coletivamente, a resolução destas lacunas e a elaboração de novos conhecimentos. ${ }^{18}$ Por meio da educação permanente abre-se a possibilidade de uma nova ação, de um novo espaço de ação e reação ${ }^{14}$, e portanto, possível, neste contexto, de trilhar-se um caminho mais seguro. 
Para reforçar a importância da atualização no setor de radiologia o Ministério da Saúde, a partir da Portaria 453, de 1998, estabelece sobre o dever das instituições prestadoras do serviço em operacionalizar programas de educação em saúde, pelo menos anualmente. Esta mesma resolução define alguns assuntos que devem ser socializados, tais como procedimentos de operações de equipamentos, uso adequado dos dosímetros individuais, uso de EPI tanto para o trabalhadores, como para paciente e acompanhantes, entre outros relacionados a segurança do setor, ${ }^{15}$ o que está sendo negligenciado nos serviços, conforme os respondentes.

É necessário que instituições que possuem trabalhadores em contato com radiação ionizante facilitem o acesso dos trabalhadores a cursos, bem como disponibilizem materiais educativos e atualizados por profissionais competentes e habilitados nessa área de conhecimento. Este recurso pedagógico pode resultar em boas práticas de segurança radiológica. ${ }^{14}$

Além disso, a informática traz consigo o ingresso de dispositivos que permitem a obtenção de imagens radiológicas digitais, sem a utilização de filme radiológico, e que associada às envergaduras dos atuais sistemas de computadores e a outros meios, redefinem a atividade radiológica, exigindo atualização constante para a adaptabilidade dos técnicos em radiologia às novas tecnologias. ${ }^{19}$ Infrações técnicas ou operacionais ocorrem, principalmente pelo desconhecimento da legislação, pela falta de um programa de manutenção preventiva dos equipamentos e da ausência de investimentos em educação e/ ou cursos de atualização profissional. ${ }^{20}$

Constata-se a importância das empresas captarem essas necessidades apresentadas pelos trabalhadores e criarem métodos, coletivamente, para determinar os rumos a serem tomados de forma a construírem ambientes saudáveis. A partir destes conhecimentos, o sucesso e qualidade de assistência aperfeiçoam-se e os danos ocupacionais são evitados.

Sobre sugestões de temas de educação em saúde, apenas $50 \%$ dos sujeitos os indicaram. Os temas mencionados pelos trabalhadores envolvem principalmente questões relativas a direitos trabalhistas e aspectos legais da profissão. A segurança, propriamente dita, não foi referida como tema principal de interesse, embora algumas respostas a permeassem.

Espaços democráticos para discussão do cotidiano entre gestores e trabalhadores e par- cerias efetivas entre as vigilâncias em saúde do trabalhador e sanitária dos estados e municípios podem auxiliar na criação e/ou manutenção de ambiências saudáveis no trabalho e garantir a segurança do trabalhador.

\section{CONCLUSÕES}

Mediante a análise dos dados apresentados, pode-se inferir que a saúde dos trabalhadores em radiologia da amostra pesquisada é vulnerável a riscos. Embora as doenças tenham sido pouco citadas, o uso dos EPIs é negligenciado por alguns e os conhecimentos a respeito da legislação sobre a segurança no trabalho é deficitário, o que sugere a necessidade de educação permanente em saúde. Sugerem-se, como futuras pesquisas, a análise de doses absorvidas de radiação e a correlação com as doenças apresentadas ao longo da atuação do trabalhador no serviço.

É importante mencionar que este estudo não tinha o intuito de entrar no âmbito jurídico e de vigilância à saúde do trabalhador, no que tange à regulação das ações de proteção radiológica, mas, diante das falas, emergiu outra sugestão de estudo.

Como se pode perceber, o tema é um campo fértil para pesquisas, na medida em que incita mudanças nos condicionantes de saúde, doença e trabalho. Respeitar o trabalhador, otimizando proteção radiológica e oportunizando espaços de educação permanente em saúde, efetivamente, contribui para a prevenção de agravos.

A ciência de que amostras pequenas limitam a generalização dos achados esteve presente em todas as fases da construção deste projeto; porém, acredita-se que os resultados aqui apresentados são considerados relevantes quando exploram dados que podem auxiliar os serviços da região estudada a refletir sobre o assunto e investir na construção de ambientes saudáveis, tendo como protagonista o trabalhador.

\section{REFERÊNCIAS}

1. Ministério da Saúde (BR). Organização PanAmericana da Saúde no Brasil: doenças relacionadas ao trabalho: manual de procedimentos para os serviços de saúde. Brasília (DF); 2001 [acesso 2009 Out 20]. Disponível em: http:/ / www.opas.org.br/ sistema/arquivos/Saudedotrabalhador.pdf

2. Macedo HA1S, Rodrigues VMCP. Programa de controle de qualidade: a visão do técnico de radiologia. Radiol Bras [online]. 2009 Feb [acesso 2010 Dec 18]; 42(1):37-41. Disponível em: http:/ / 
www.scielo.br/scielo.php?pid=S010039842009000 $100009 \&$ script $=$ sci_arttext

3. Espíndola KKL, Ramos IC, Leitão IMTA. Medidas de prevenção e controle de infecção: percepção e conhecimento dos técnicos em radiologia. Cienc Cuid Saude 2008 Jul-Set; 7(3):311-18

4. Diniz DN, Bento PM, Pereira MSV, Pereira JV, Silva DF, Costa MRM, Albuquerque CL. Avaliação do conhecimento sobre biossegurança em radiologia pelos alunos do curso de Odontologia da Universidade Estadual da Paraíba. Arq Ciênc Saúde. 2009 Out-Dez; 16(4):166-9

5. Fernandes GS, Carvalho ACP, Azevedo ACP. Avaliação dos riscos ocupacionais de trabalhadores de serviços de radiologia. Radiol Bras [online]. 2005 Aug [acesso 2010 Dez 17]; 38(4):279-81. Disponível em: http:/ / www.scielo.br/scielo.php?script=sci_a rttext\&pid=S0100-39842005000400009

6. Ministério da Ciência e Tecnologia (BR), Cardoso EM. Programa de integração Comissão Nacional de Energia Nuclear: módulo de informação técnica [online]. [acesso 2008 Out 17]. Disponível em: http:// www.cnen.gov.br/ensino/apostilas/PIC.pdf

7. Silva MG, Fernandes JG, Teixeira GAS, Silva RMO. Processo de formação da(o) enfermeira(o) na contemporaneidade: desafios e perspectivas. Texto Contexto Enferm. 2010 Jan-Mar ; 19(1):176-84.

8. Polit DF, Beck CT, Hungler BP. Fundamentos de pesquisa em enfermagem. $5^{\text {a }}$ ed. São Paulo (SP): Artmed; 2004.

9. Conselho Nacional de Técnicos em radiologia. Lei 7394 de 29 de outubro de 1995. Regula o Exercício da profissão de Técnico em Radiologia e dá outras providências [acesso 2010 Mar 23]. Disponível em: http://www.jusbrasil.com.br/legislacao/110494/ lei-7394-85

10. Ministério da Saúde (BR). Conselho Nacional de Saúde. Diretrizes e normas regulamentadoras da pesquisa envolvendo seres humanos: Resolução no.196/96. Brasília (DF); 1996 [acesso 2009 Jun 17]. Disponível em: http://www.ufrgs.br/bioetica/ res19696.htm

11. Morais EM, Dutra LM, Fontana RT. A cefaléia e a saúde do trabalhador de enfermagem: análise de uma realidade. In: Anais do II Fórum Internacional Integrado de Cidadania; 2009. Santo Ângelo/RS Universidade Regional Integrada do Alto Uruguai e das Missões - Campus Santo Ângelo; 2009.

12. Ministério do Trabalho e Emprego (BR). Norma Regulamentadora $n^{\circ}$ 7: dispõem sobre o Programa de
Controle Médico de Saúde Ocupacional (PCMSO): Portaria SSST n. ${ }^{\circ 24}$, Brasília (DF); 1994 [acesso 2010 Mai 27]. Disponível em: http:/ / www.mte.gov.br/ legislacao/normas_regulamentadoras/default.asp

13. Ministério do Trabalho e Emprego (BR). Norma Regulamentadora $n^{\circ} 6$. dispõem sobre Equipamento de Proteção Individual (EPIs): Portaria GM n. ${ }^{\circ} 3.214$ Brasília (DF); 1978 [acesso 2010 Jun 02]. Disponível em: http://www.mte.gov.br/legislacao/normas regulamentadoras/default.asp

14. Flôr RC, Gelbcke FL. Tecnologias emissoras de radiação ionizante e a necessidade de educação permanente para uma práxis segura da enfermagem radiológica. Rev Bras Enferm. 2009 Oct; 62(5):766-70.

15. Ministério da Saúde (BR). Portaria 453. Diretrizes de proteção radiológica e radiodiagnóstico médico e odontológico. Brasília: Secretaria de Vigilância Sanitária, Ministério da Saúde; 1998.

16. Ministério da Ciência e da Tecnologia (BR). Comissão Nacional de Energia Nuclear. Diretrizes básicas de proteção radiológica: Norma CNEN NN-3.01. Brasília (DF); 2005 [acesso 2009 Out 17]. Disponível em: www.cnen.gov.br/seguranca/ normas/pdf/Nrm301.pdf

17. Oliveira SR, Azevedo ACP, Carvalho ACP. Elaboração de um programa de monitoração ocupacional em radiologia para o Hospital Universitário Clementino Fraga Filho. Radiol Bras [online]. 2003 Jan [acesso 2010 Dec 18]; 36(1):27-34. Disponível em: http://www.scielo.br/scielo. php?script=sci_arttext\&pid $=\$ 010039842003000100$ 008\&lang=pt

18. Ministério da Saúde (BR). Gabinete do Ministro. Diretrizes Para a implementação da Política Nacional de Educação em Saúde Permanente e dá outras providências: Portaria n ${ }^{\circ} 1996$. Brasília (DF); 2007. [acesso 2008 Out 20]. Disponível em: http:// portal.saude.gov.br/portal/saude/profissional/ area.cfm?id_area $=1265$

19. Felício CMF, Rodrigues VMCP. A adaptação do técnico de radiologia às novas tecnologias. Radiol Bras. 2010 Fev [citado 2010 Jun 03]; 43(1): 23-8.

20. Pacheco JG, Santos MB, Tavares-Neto J. Avaliação dos serviços de radiodiagnóstico convencional de dois hospitais da rede pública estadual de Rio Branco, Acre. Radiol Bras [online]. $2007 \mathrm{Fev}$ [acesso 2010 Dez 18]; 40(1):39-44. Disponível em: http://www. scielo.br/scielo.php?script $=$ sci_arttext\&pid $=\mathrm{S} 0100-$ 39842007000100010\&lng=pt\&nrm=iso 